\title{
Design of near zero ultra-flattened chromatic dispersion highly non linear holey fiber in tele communication band
}

\author{
Mahbubur Rahman*, A.H. Md. Mostazir, M. A. Alam, Md. Samiul Habib \\ Department of Electrical \& Electronic Engineering, Rajshahi University of Engineering \& Technology, Rajshahi-6204, Bangladesh \\ *Corresponding author E-mail: engr.rumman@gmail.com
}

\begin{abstract}
We propose a four-ring hexagonal holey fiber (HF) which exhibits near zero ultra-flattened chromatic dispersion and nonlinear property simultaneously in a modest number of rings. The finite element method with perfectly matched layers boundary condition is used to investigate the guiding properties. A four ring HF with flattened dispersion of $0.85 \mathrm{ps} / \mathrm{nm} / \mathrm{km}$ from 1.14 to $1.60 \mu \mathrm{m}$ wavelength range, $21.34 \mathrm{~W}-1 \mathrm{~km}-1$ nonlinear coefficient and splice loss $3.82 \mathrm{~dB}$ at $1.55 \mu \mathrm{m}$ is numerically demonstrated.
\end{abstract}

Keywords: Finite element method, Holey Fiber, Nonlinear fiber, splice loss, Ultra-flattened Chromatic Dispersion.

\section{Introduction}

Holey fibers (HFs) [1] consisting of a central defect region surrounded by air holes running parallel to the fiber length have been one of the most interesting development in recent fiber optics [2]. HFs has emerging applications such as power delivery ultra-short pulse delivery, and pulse compression require refining and designing other characteristics, most notably increased bandwidth, near-zero flat chromatic dispersion, and control of the nonlinear responses [3]. Nowadays, highly nonlinear Holey Fibers [1] (HNL-HFs) has finding applications in all-optical signal processing, wavelength converter, ultra-short soliton pulse transmission, optical parametric amplification, and super continuum generation [4]. HFs provide higher nonlinear interaction over a significant length of fibers even with a moderate input power level [5]. Due to this important reason HNL-HF is important in research applications.

We, in this paper, propose a four-ring hexagonal holey fiber which exhibits the flat dispersion and nonlinear property simultaneously. Many HFs design exists in the literature with remarkable dispersion and leakage properties but nonlinear coefficient of HFs are often less than a $16 \mathrm{~W}-1 \mathrm{~km}-1$ [5] and the fiber having nonlinear coefficient greater than this value is treated as highly nonlinear fiber. Only two variable parameters are used in the proposed HFs. We obtain ultra-flattened dispersion of $0.85 \mathrm{ps} / \mathrm{nm} / \mathrm{km}$ over the band 1.14 to $1.60 \mu \mathrm{m}$ and nonlinear coefficient of the order of 21.34 W-1Km-1from the proposed HOF. Due to lesser number of air-hole and shortest varying parameter the fabrication of the proposed fiber will be easier. Abdur Razzak et al. [3] proposed HNL-HF with 200nm dispersion flat whereas our proposed HNL-HF exhibits 460nm flat dispersion.

\section{Design methodology of the proposed DF-HNFs}

Consider the cross-section of the simple four ring structured highly nonlinear dispersion flattened Holey fiber (DF-HF) as shown in Fig. 1. The cladding consists of air holes embedded in silica and the air hole pitch is labeled $\Lambda$ (the distance between the centers of neighboring air holes). The cladding is formed by an equilateral triangular lattice of air holes. We use four rings of air holes surrounding the core. Two types of air-hole with diameters $d_{l}$ and $d$ is introduced. Here, the first air-hole diameter is $d_{l}$ and the second, third and fourth ring diameter is same and is denoted by $d$. The refractive index of fiber silica is $n_{s}=1.4457$ and refractive index of air-hole is $n_{a}=1$. The major challenge associated with designing highly nonlinear HFs is setting the zero dispersion wavelength around the telecom window because a HF with a short pitch and uniform smaller air-hole dimensions tend to shift the zero-dispersion wavelength towards shorter wavelengths and a HF with higher air-hole dimension relative to the pitch sets a limit on the single mode operation bandwidth [4]. 
In index-guiding HFs, since the periodicity in the cladding region is not essential to confine the guiding light into the high-index core region, this paper is followed this design principle to control the dispersion and dispersion slope in wide wavelength range. The challenge exists to minimize confinement loss and control of the sensitivity to parameter variations. In fig. 1 there are three degrees of freedom for controlling dispersion behavior and nonlinear property of four rings HNF, namely $d_{l}, d\left(d_{l}<d\right)$ and $\Lambda$, the diameter of first ring is kept low to obtain near zero flat dispersion and diameter of the outer three rings are kept large for better field confinement and for reducing confinement loss. We have shown theoretically that it possible to design a simple HNF with reduced air-hole without distorting the dispersion flatness in telecommunication band.

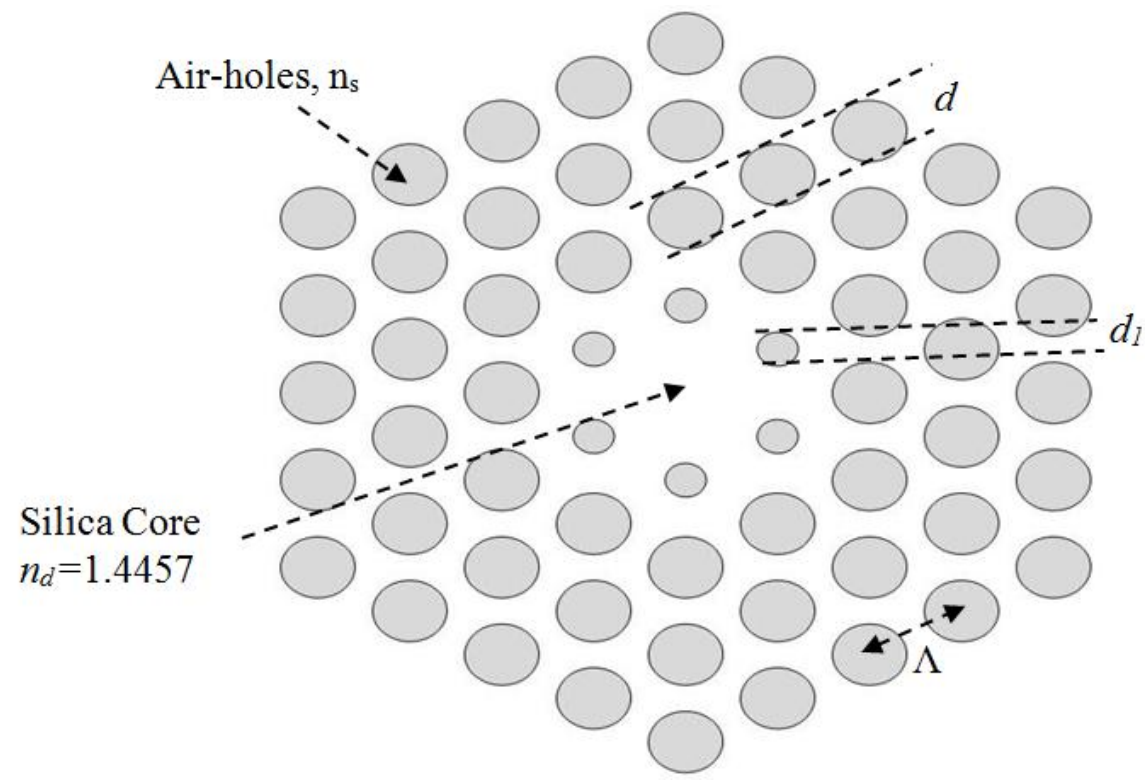

Fig. 1: Proposed Geometrical structure of four rings DF-HNF. Number of rings, $\mathrm{N}_{\mathrm{r}}=4$.

\section{Simulation technique}

The COMSOL 4.2 and MATLAB 2008a version software are used as a simulation tool. Effective refractive index, effective area, chromatic dispersion and confinement loss are calculated by using finite element method (FEM) with perfectly matched boundary layers (PML). FEM has been combined with most of the other computational methods to develop more efficient hybrid computational methods. The FEM allows the MOF cross-section in the transverse $x-y$ plane to be divided into a patchwork of triangular elements, which can be of different sizes, shapes and refractive indices. FEM is suited for studying fibers with no periodic air-hole arrangements [6]. The FEM directly solves the Maxwell equations to best approximate the value of the effective refractive index. However, the modal effective refractive index, $\mathrm{n}_{\mathrm{eff}}$ is obtained by solving an Eigen value problem drawn from Maxwell equations using the COMSOL 4.2 software, effective area $\mathrm{A}_{\text {eff }}$ can be obtained using the following equation [7].

The effective area $A_{\text {eff }}$ is calculated by

$$
\mathrm{A}_{\mathrm{eff}}=\left(\int_{-\infty}^{+\infty} \int_{-\infty}^{+\infty}|E|^{2} \mathrm{dxdy}\right)^{2} / \int_{-\infty}^{+\infty} \int_{-\infty}^{+\infty}|E|^{2} \mathrm{dx} \mathrm{dy}
$$

Where, $\mathrm{E}$ is the electric field derived by solving the Maxwell equations.

Chromatic dispersion D can be calculated by the relations [8].

$$
\mathrm{D}(\lambda)=-\lambda / \mathrm{c}(\mathrm{d} 2 \operatorname{Re}[\text { neff }] / \mathrm{d} \lambda 2
$$

Where $\operatorname{Re}\left[\mathrm{n}_{\mathrm{eff}}\right]$ is the real part of effective refractive index, $\mathrm{n}_{\mathrm{eff}}, \lambda$ is the wavelength, $\mathrm{c}$ is the velocity of light in vacuum. The material dispersion given by Sellmeier formula is directly included in the calculation. Therefore, D in (2) corresponds to the chromatic dispersion of the DF-HNF.

The confinement loss $\mathrm{L}_{\mathrm{c}}$ is obtained from the imaginary part of $\mathrm{n}_{\mathrm{eff}}$ as follows [8] 


$$
\mathrm{Lc}=8.686 \times \mathrm{k} 0 \mathrm{Im}[\mathrm{neff}]
$$

Where $\operatorname{Im}\left[\mathrm{n}_{\mathrm{eff}}\right]$ is the imaginary part of the refractive index, $\mathrm{k}_{0}=2 \pi / \lambda$ is the wave number in the free space.

\section{Simulation results}

In this proposed DF-HNF design, optimizing the geometrical parameters, such as air hole diameters and pitch, the ultraflattened near zero dispersion with high nonlinear coefficient can be efficiently designed. We first set air-hole diameters of the outer cladding at $d / \Lambda=0.7$ for Fig. 2. In the outer most cladding a high value air filling fraction is chosen for better field confinement. Dimension of the first ring is lowered down to flatten the dispersion characteristics. As shown in Fig. 1, there are three tuning parameters $d_{l}, d$ and $\Lambda$ for shaping the dispersion behavior of desired shape. We carefully investigated the effects of $d_{l} / \Lambda, d / \Lambda$ and pitch, $\Lambda$ on dispersion behavior which has been shown later.

\subsection{Effect of changing $d_{1} / \Lambda$ on the dispersion slope}

Suitable normalized air-hole diameter of first ring $d_{l} / \Lambda$ is found by calculating the dispersion as a function of wavelength for several different $d_{1} / \Lambda$. Fig. 2 shows an example of the effect of changing $d_{1} / \Lambda$ on the dispersion slope with $d_{l} / \Lambda=0.385,0.386,0.387,0.388$ respectively, for a fixed air-hole pitch $\Lambda=1.15 \mu \mathrm{m}$ and outer ring air filling fraction $d / \Lambda=0.7$. From fig. 2 , it has been shown that flattened dispersion greatly depends on first ring air-filling fraction $d_{l} / \Lambda$. if the first ring air-filling fraction raises the slope changes significantly from the positive slope to the negative slope near the wavelength $1.20 \mu \mathrm{m}$.

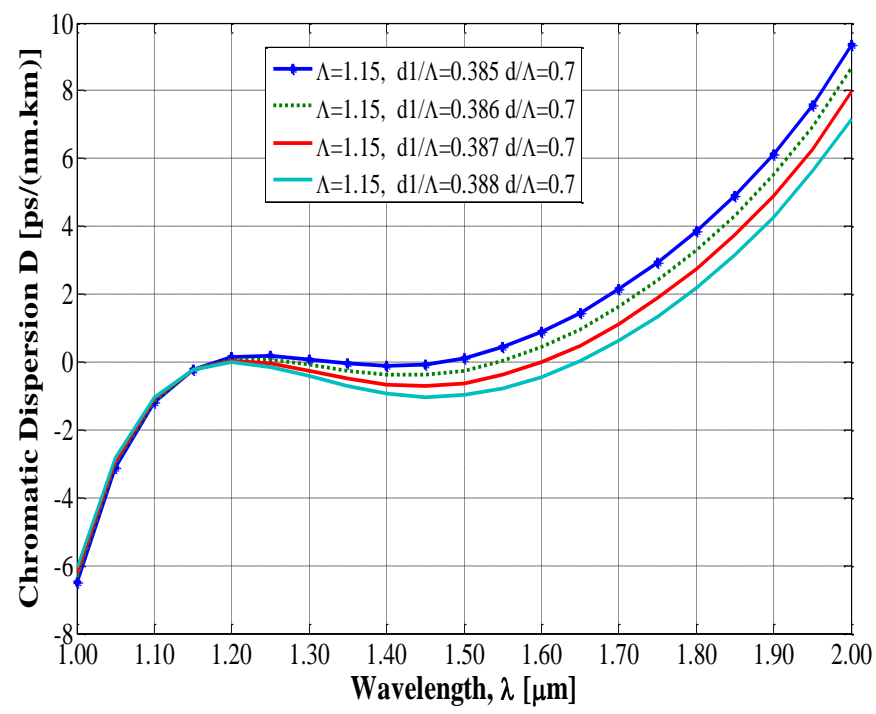

Fig. 2: Effect on dispersion due to $d_{l} / \Lambda$ variation with $d_{l} / \Lambda=0.385,0.386,0.387$ and 0.388 .

\subsection{Effect of changing $d / \Lambda$ on the dispersion level}

Suitable normalized air-hole diameter of outer ring $d / \Lambda$ is found by calculating the dispersion as a function of wavelength for several different $d / \Lambda$. Fig. 3 shows an example of the effect of changing $d / \Lambda$ on the dispersion slope with $d / \Lambda=0.7,0.8$ and 0.9 respectively.

Fig. 3 shows the effect of changing $d / \Lambda$ on dispersion level with $d / \Lambda=0.6,0.7$ and 0.8 respectively for a fixed air-hole pitch $\Lambda=1.15$ and $d_{l} / \Lambda=0.386$. It has seen that there is a large impact on variation of slope for variation of outer ring diameter. Here, optimum dispersion is obtained at $\Lambda=1.15, d_{1} / \Lambda=0.386$ and $d / \Lambda=0.7$. 


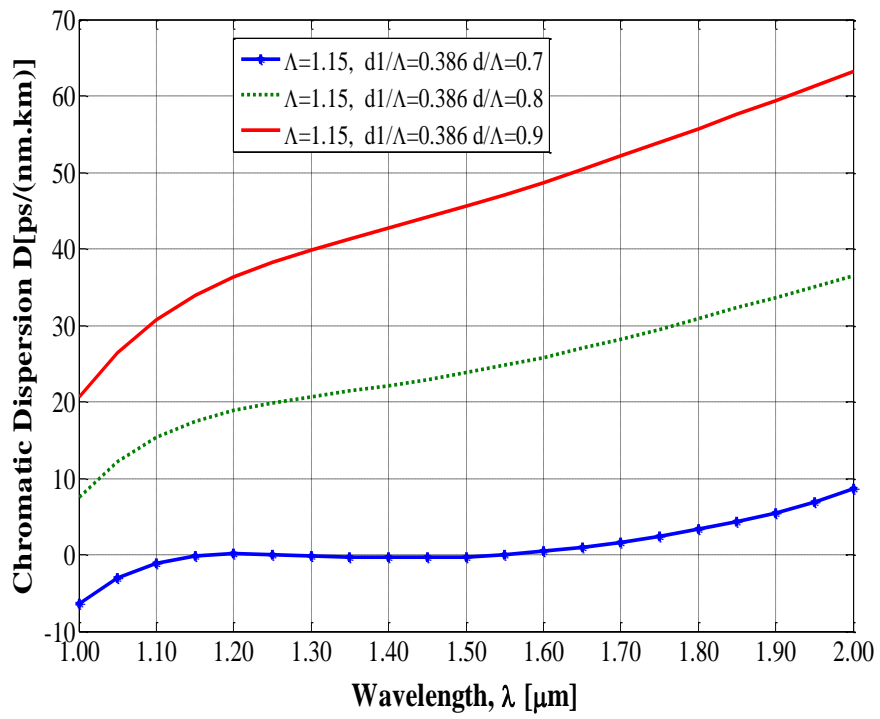

Fig. 3: Effect on dispersion due to $d / \Lambda$ variation with $d / \Lambda=0.7,0.8$ and 0.9 .

\subsection{Effect of changing pitch $\Lambda$ on the dispersion level}

Fig. 4 shows the pitch variation $\Lambda$ on the dispersion level. Generally pitch dominantly influence the dispersion level but little impact on the slope of dispersion but in case of HNF dispersion behavior is too much sensitive to the pitch. Fig. 4 shows the effect of changing air-hole pitch $\Lambda$ on the chromatic dispersion behavior with $d_{1} / \Lambda=0.86, d / \Lambda=0.9$. Air-hole pitch is varied to achieve target zero ultra-flattened chromatic dispersion in wide-band wavelength range.

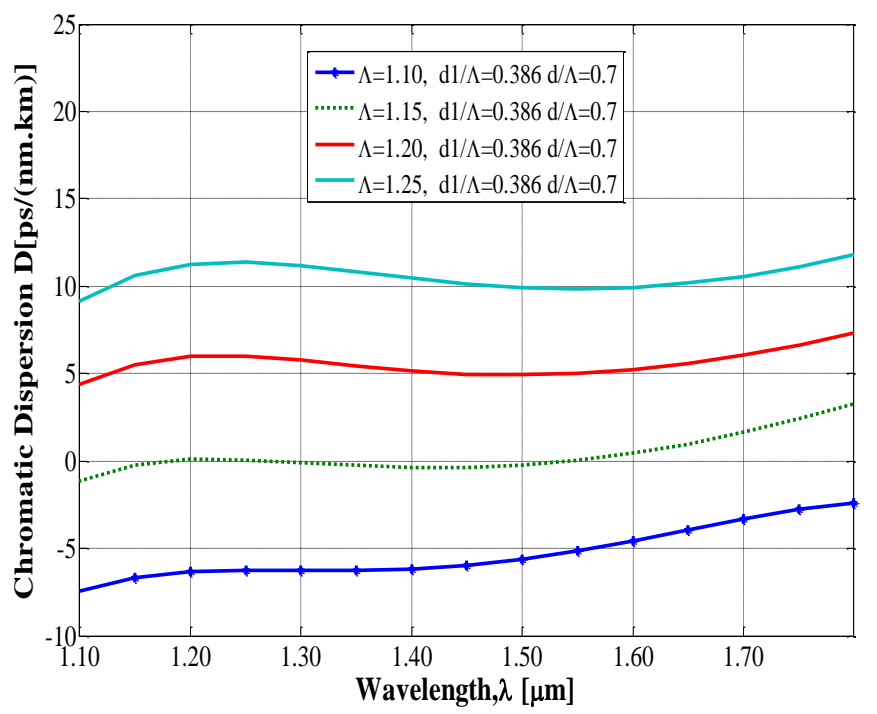

Fig. 4: Effect on dispersion due to $\Lambda$ variation with $\Lambda=1.00,1.15,1.20$ and $1.25 \mu \mathrm{m}$.

\subsection{Nearly-Zero ultra-flattened dispersion}

For optimum result, air-hole diameter on the first ring is scaled down to shape dispersion property while diameter of airholes on outer rings is kept larger for better field confinement. Finally, air-hole pitch, $\Lambda$ is varied to achieve target zero ultra-flattened chromatic dispersion in wide band wavelength range.

Suitable normalized air-hole diameter of first ring $d_{l} / \Lambda$ is found by calculating the dispersion as a function of wavelength for several different $d_{l} / \Lambda$. We have shown in fig. 2 that flattened dispersion greatly depends on first ring airfilling fraction $d_{l} / \Lambda$. if the first ring air-filling fraction raises the slope changes significantly from the positive slope to the negative slope near the wavelength $1.20 \mu \mathrm{m}$. 


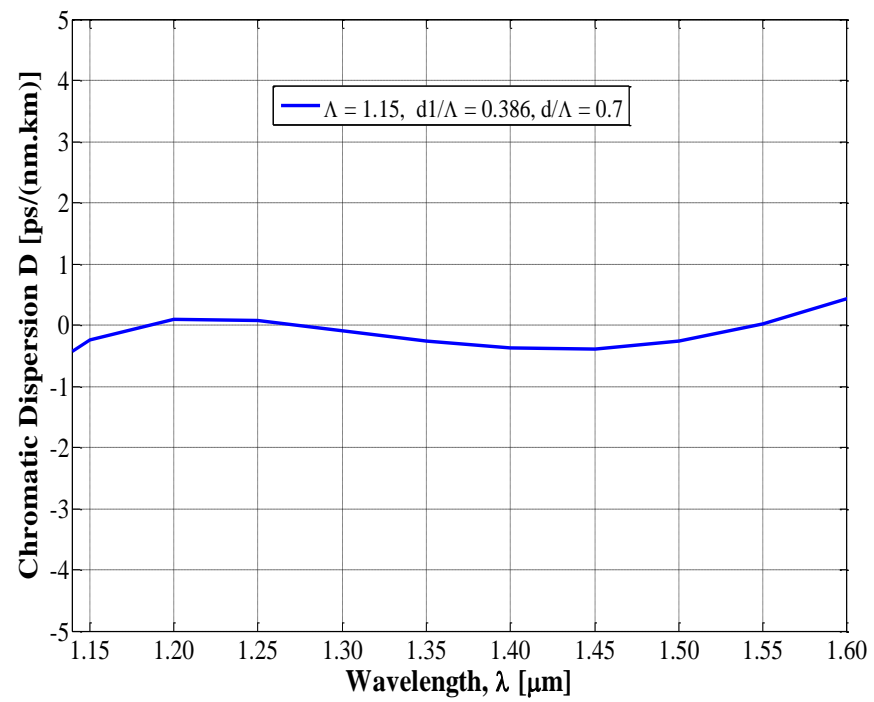

Fig. 5: Wavelength dependence of the DF-HNF's chromatic dispersion for optimum parameters: $d_{l} / \Lambda=0.386, d / \Lambda=0.7, \Lambda=1.15 \mu \mathrm{m}$ and $\mathrm{N}_{\mathrm{r}}=4$.

\subsection{Effect of nonlinear co-efficient:}

The fiber is said to be highly nonlinear fiber, if nonlinear coefficient is greater than $16 W^{-1} \mathrm{Km}^{-1}$ at $1.55 \mu \mathrm{m}$ wavelength. Fig. 6 shows the nonlinear coefficient of the HNL-HF with respect to wavelength. We have found from the fig. 6 that the nonlinear coefficient of the HNL-HF at $1.55 \mu \mathrm{m}$ is $21.34 \mathrm{~W}^{-1} \mathrm{Km}^{-1}$.

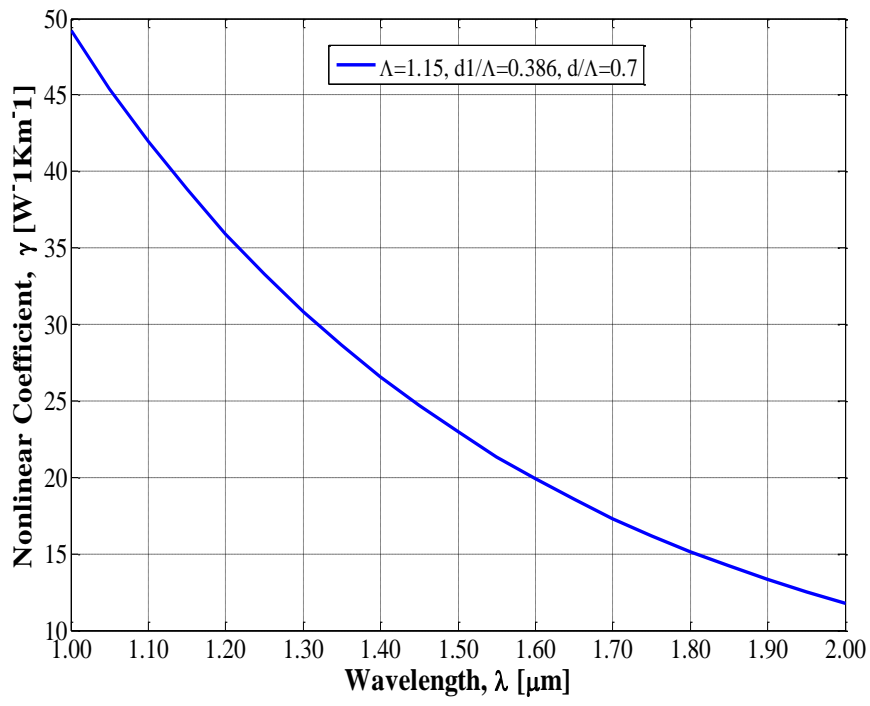

Fig. 6: Wavelength dependence of nonlinear co efficient of the DF-HNF for Optimum parameters: $d_{l} / \Lambda=0.386, d / \Lambda=0.7, \Lambda=1.15 \mu \mathrm{m}$ and $\mathrm{N}_{\mathrm{r}}=4$.

\subsection{Effect of confinement loss}

In this proposed DF-HNF design confinement loss at $1.55 \mu \mathrm{m}$ wavelength is the order of $10^{2} \mathrm{~dB} / \mathrm{km}$, which is very large. This large confinement loss is undesirable. This large confinement loss occurs due to small size of outer ring diameter. By increasing number of ring or using air hole in octagonal structure instead of hexagonal structure, confinement loss can be efficiently minimized. Increasing ring number introduces fabrication challenge. Graph shows wavelength dependence on confinement loss of the proposed DF-HNF's at 1550nm wavelength for optimum design parameters. Fig. 7 shows the confinement loss of the HNL-HF corresponding to $d_{1} / \Lambda=0.386 \mu \mathrm{m}, d / \Lambda=0.7 \mu \mathrm{m}$. Confinement loss is increasing smoothly with respect to wavelength and there is no evidence of abrupt change in leakage. Increasing losses due to corresponding decrease in air-hole diameters are also consistent. 


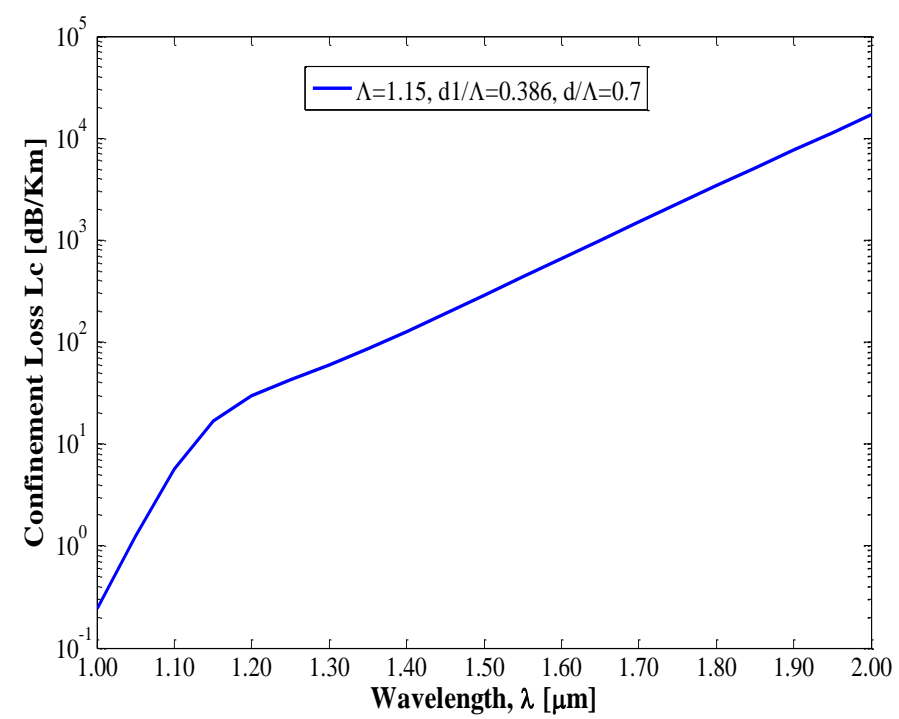

Fig. 7: Wavelength dependence of the DF-HNF's confinement loss for optimum parameters: $d_{1} / \Lambda=0.35, d_{2} / \Lambda=0.38, d / \Lambda=0.9, \Lambda=1.63 \mu \mathrm{m}$ and $\mathrm{N}_{\mathrm{r}}=4$.

\subsection{Wavelength dependence of effective area}

Fig. 8 shows effective areas of the fiber for optimum design parameters. The effective area of the proposed DF-HNF's is obtained from optimum design parameters: $d_{1} / \Lambda=0.386, d / \Lambda=0.7, \Lambda=1.15 \mu \mathrm{m}$ and $\mathrm{N}_{\mathrm{r}}=4$. If outer ring diameter increases, the effective area of the proposed HNF decreases. Again, similarly the effective area of the proposed HNF decreases according to first ring diameter increases. The effective area of the proposed fiber at $1.55 \mu \mathrm{m}$ is approximately $4.4 \mu \mathrm{m}^{2}$.

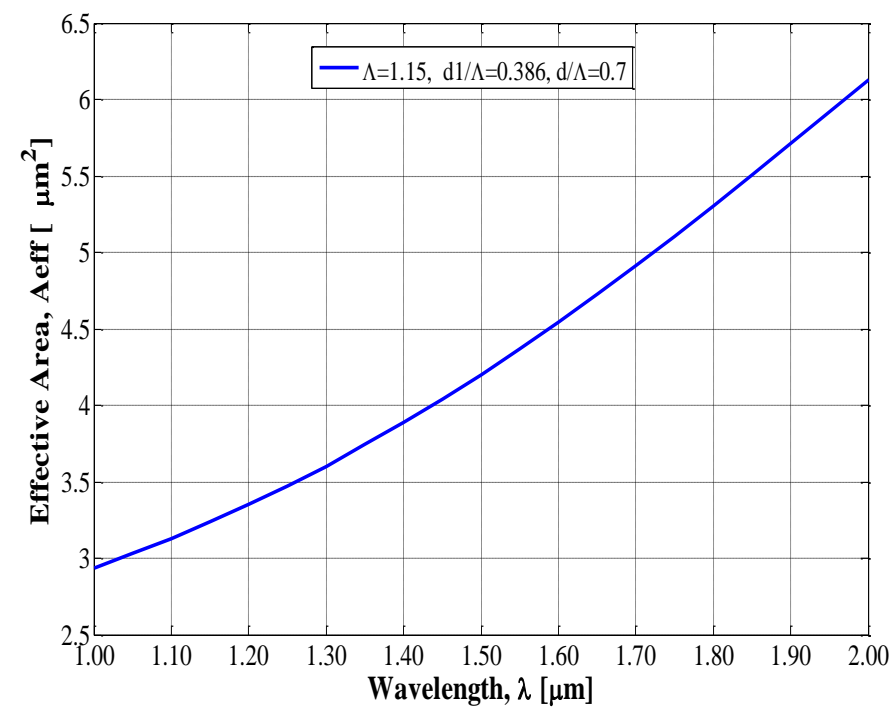

Fig. 8: Wavelength dependence of the DF-HNF's effective area for optimum parameters: $d_{1} / \Lambda=0.386, d / \Lambda=0.7, \Lambda=1.15 \mu \mathrm{m}$ and $\mathrm{N}_{\mathrm{r}}=4$.

\subsection{Effect of splice loss}

Fig. 9 shows wavelength dependence of mode field diameter (MFD) and splice loss between this fiber and conventional single mode fibers (SMFs). The MFD of the SMF is considered $10.0 \mu \mathrm{m}$. The MFD is calculated by the well-known pitermann II formula [9], and the splice loss Ls is calculated by [10]-

$$
L_{s}=-20 \log _{10} \frac{2 w_{S M F} w_{H F}}{w_{S M F}^{2}+w_{H F}^{2}}
$$


Where, $\mathrm{W}_{S M F}$ and $\mathrm{W}_{H F}$ are the mode field diameters of the SMF and the HF respectively. MFD and Splice loss of proposed fiber is $3.65 \mu \mathrm{m}$ and $3.82 \mathrm{~dB}$ at $1.55 \mu \mathrm{m}$.

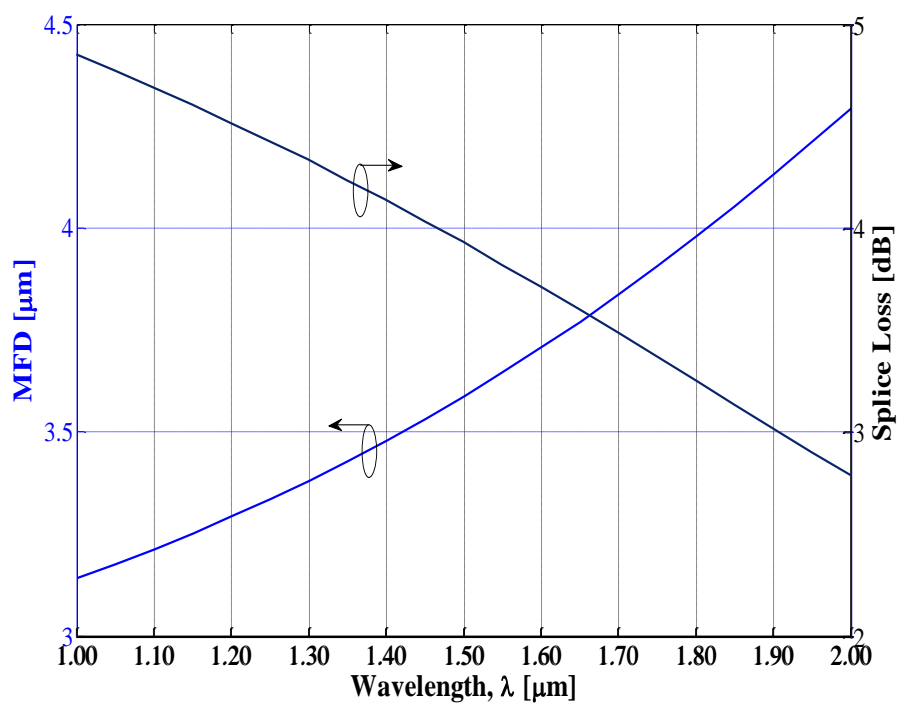

Fig. 9: Wavelength dependence of the DF-HNF's Splice loss and MFD for optimum parameters: $d_{l} / \Lambda=0.386, d / \Lambda=0.7, \Lambda=1.15 \mu \mathrm{m}$ and $\mathrm{N}_{\mathrm{r}}=4$.

\section{Conclusion}

A truly near zero ultra-flattened dispersion HNF has been proposed in simple design. This fiber has a modest number of design parameters, four rings, three air-hole diameters, and a common air-hole pitch. It has been shown through numerical simulation results that a four-ring DF-HNF can assume nearly zero ultra-flattened dispersion of $0.85 \mathrm{ps} / \mathrm{nm} / \mathrm{km}$ in a $1.14 \mu \mathrm{m}$ to $1.6 \mu \mathrm{m}$ (460 nm bandwidth) wavelength range with high nonlinear coefficient. The main drawback of our proposed HNF is high confinement loss. Fibers having nearly zero ultra-flattened chromatic dispersion and high nonlinear coefficient are very crucial for broadband communication systems.

We have demonstrated how a smart utilization of the geometry of the photonic crystal cladding of a hexagonal HF permits an outstanding control of the dispersion properties of the fiber. The fact that the geometrically-induced dispersion of a HF has remarkable properties and it is highly tunable in terms of the geometrical parameters of the fiber can be used to properly compensate the inherent dispersion of the silica in many different ways. The key point is the understanding of the interplay between both type of dispersions, which is easily achieved by means of a suitable graphical representation and the use of the approximate equation for the total dispersion. With further optimization of the structure and better control of the fluctuations in fiber diameter, we expect these dispersion values to be reduced still further.

\section{References}

[1] J. C. Knight, T. A. Birks, P. St. J. Russell, and D. M. Atkin, “All-silica single- mode optical fiber with photonic crystal cladding,” Opt. Lett., vol. 21, pp.1547-1549 Oct.1996.

[2] Feroza Begum et al, "Design and analysis of novel highly nonlinear photonic crystal fibers with ultra-flattened chromatic dispersion", Optics Communications 282 (2009) 1416-1421.

[3] S. M. Abdur Razzak, Yoshinori Namihira, "Proposal for Highly Nonlinear Dispersion-Flattened Octagonal Photonic Crystal Fibers", IEEE Photonics Technology Letters, Vol. 20, No. 4, February 15, 2008.

[4] Photonic Crystal Fibers: Design and Applications S. M. Abdur Razzak (Author), Yoshinori Namihira (Author).

[5] S. M. Abdur Razzak et al, "Optimum Design of a Dispersion Managed Photonic Crystal Fiber for Nonlinear Optics Applications in Telecom Systems", 5th International Conference on Electrical and Computer Engineering ICECE 2008, 20-22 December 2008, Dhaka, Bangladesh.

[6] T. M. Monro, D. J. Richardson, N. G. R. Broderick, and P. J. Bennett, "Modeling large air fraction holey optical fibers," IEEE/OSA Journal of Lightwave Technology, vol. 18, pp. 50-56, Jan. 2000.

[7] S. M. A. Razzak, Y. Namihira, F. Begum, S. Kaijage, N. H. Hai, and N. Zou, "Design of a decagonal photonic crystal fiber with ultra-flattened chromatic dispersion,” IEICE Trans. Electron., vol. E90-C, no. 11, pp. 2141-2145, Nov. 2007.

[8] K. Saitoh, M. Koshiba, T. Hasegawa, E. Sasaoka, "Chromatic dispersion control in photonic crystal fibers: application to ultra-flattened dispersion,” Opt. Express, vol. 11, no. 8, pp.843-852, April 2003.

[9] K. Petermann, "Contrainsts for fundamental-mode spot size for broadband dispersion-compensated single-mode fibers," Electron. Lett., vol. 19, pp. 712-714, September 1983.

[10] F. Zolla, G. Renversez, A. Nicolet, B. Kuhlmey, S. Guenneau, D. Felbacq, Foundation of Photonic Crystal Fibres, Imperial Collage Press, London, 2005. 